David Gérard-Varet · Nader Masmoudi

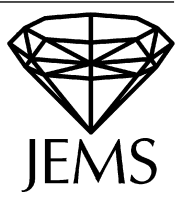

\title{
Homogenization in polygonal domains
}

Received October 8, 2008 and in revised form June 21, 2010

\begin{abstract}
We consider the homogenization of elliptic systems with $\varepsilon$-periodic coefficients. Classical two-scale approximation yields an $O(\varepsilon)$ error inside the domain. We discuss here the existence of higher order corrections, in the case of general polygonal domains. The corrector depends in a non-trivial way on the boundary. Our analysis substantially extends previous results obtained for polygonal domains with sides of rational slopes.
\end{abstract}

\section{Introduction}

This paper is devoted to elliptic systems in divergence form, with Dirichlet boundary condition:

$$
\left\{\begin{array}{rlrl}
-\nabla \cdot A(x / \varepsilon) \nabla u^{\varepsilon} & =f, & & x \in \Omega, \\
u^{\varepsilon} & =0, & x \in \partial \Omega,
\end{array}\right.
$$

set in a bounded domain $\Omega \subset \mathbb{R}^{d}$. For simplicity, we assume $d=2$ or 3 . Following standard notation, $\varepsilon>0$ is a small parameter, and $A=A^{\alpha \beta}(y) \in M_{n}(\mathbb{R})$ is a family of functions of $y \in \mathbb{R}^{d}$ with values in the set of $n \times n$ matrices, indexed by $1 \leq \alpha, \beta \leq d$. The unknown and source term are $u^{\varepsilon}=u^{\varepsilon}(x) \in \mathbb{R}^{n}$ and $f=f(x) \in \mathbb{R}^{n}$. We recall, using Einstein's summation convention, that for each $1 \leq i \leq n$,

$$
(\nabla \cdot A(x / \varepsilon) \nabla u)_{i}:=\partial_{x_{\alpha}}\left[A_{i j}^{\alpha \beta}(x / \varepsilon) \partial_{x_{\beta}} u_{j}\right] .
$$

We assume that $A$ and $f$ are smooth. Finally, we make the following hypothesis:

(i) Ellipticity: For some $\lambda>0$, for all families of vectors $\xi=\xi^{\alpha} \in \mathbb{R}^{n}$ indexed by $1 \leq \alpha \leq d$

$$
\lambda \xi^{\alpha} \cdot \xi^{\alpha} \leq A \xi \cdot \xi \leq \lambda^{-1} \xi^{\alpha} \cdot \xi^{\alpha}
$$

where

$$
A \xi \cdot \xi:=\sum_{\alpha, \beta, i, j} A_{i j}^{\alpha, \beta} \xi_{j}^{\beta} \xi_{i}^{\alpha}
$$

D. Gérard-Varet: IMJ, Université Denis Diderot, 175 rue du Chevaleret, 75013 Paris, France; e-mail: gerard-varet@math.jussieu.fr

N. Masmoudi: Courant Institute of Mathematical Sciences, 251 Mercer Street, New York, NY 10012, USA; e-mail: masmoudi@ courant.nyu.edu 
(ii) Periodicity:

$$
A(y+h)=A(y), \quad \forall y \in \mathbb{R}^{d}, \forall h \in \mathbb{Z}^{d} .
$$

We are interested in the limit $\varepsilon \rightarrow 0$, i.e. the homogenization of system (1.1).

System (1.1) is a classical model from linear elasticity theory. It describes the deformation of a solid object, made of a composite material. The microstructure of the composite material is modeled by the matrix $A$, with characteristic length $\varepsilon$. The field $u^{\varepsilon}$ describes the displacement of the solid when subjected to a body force $f$. The homogenization of equation (1.1) aims at simplifying the microscopic description, by extracting some macroscopic averaged properties. It has given birth to a rich mathematical theory (see the articles [14, 19] and textbooks [11, 8], among many).

As a special case, periodic homogenization has a very long history, and we refer to the classical book [6]. The starting point of most studies is a formal two-scale expansion of the solution $u^{\varepsilon}$,

$$
u^{\varepsilon}=u^{0}(x)+\varepsilon u^{1}(x, x / \varepsilon)+\varepsilon^{2} u^{2}(x, x / \varepsilon)+\cdots .
$$

The leading term $u^{0}$ satisfies the homogenized system

$$
\left\{\begin{aligned}
-\nabla \cdot A^{0} \nabla u^{0} & =f, & & x \in \Omega, \\
u^{0} & =0, & & x \in \partial \Omega .
\end{aligned}\right.
$$

The homogenized matrix $A^{0}$ comes from the averaging of the microstructure. It involves the periodic solution $\chi=\chi^{\gamma}(y) \in M_{n}(\mathbb{R}), 1 \leq \gamma \leq d$, of the famous cell problem:

$$
-\partial_{y_{\alpha}}\left[A^{\alpha \beta}(y) \partial_{y_{\beta}} \chi^{\gamma}(y)\right]=\partial_{y_{\alpha}} A^{\alpha \gamma}(y), \quad \int_{[0,1]^{d}} \chi^{\gamma}(y) d y=0 .
$$

More precisely $A^{0}$ is given by

$$
A^{0, \alpha \beta}=\int_{[0,1]^{d}} A^{\alpha \beta}+\int_{[0,1]^{d}} A^{\alpha \gamma} \partial_{y_{\gamma}} \chi^{\gamma} .
$$

The second term in the expansion (1.2) reads

$$
u^{1}(x, y):=\tilde{u}^{1}(x, y)+\bar{u}^{1}(x):=-\chi^{\alpha}(y) \partial_{x_{\alpha}} u^{0}(x)+\bar{u}^{1}(x),
$$

where $\chi$ is again the solution of (1.4).

All profiles $u^{k}=u^{k}(x, y)$ in (1.2) are periodic in $y$, and therefore do not satisfy the homogeneous Dirichlet boundary condition. However, the first terms of the expansion are relevant, and the following bound holds (see [6]):

$$
\left\|u^{\varepsilon}-u^{0}(x)-\varepsilon u^{1}(x, x / \varepsilon)\right\|_{H^{1}(\Omega)}=O(\sqrt{\varepsilon}) .
$$

It is known that this estimate is optimal: as the approximation is not zero at the boundary, there is a boundary layer phenomenon, responsible for an $O(\sqrt{\varepsilon})$ loss in (1.6). However, if a relatively compact subset $\omega \Subset \Omega$ is considered, one may avoid this loss, as strong 
gradients near the boundary are filtered out. Precisely, Avellaneda and Lin prove in [5], under some regularity assumptions on $A$ and $\Omega$, that

$$
\left\|u^{\varepsilon}-u^{0}(x)-\varepsilon u^{1}(x, x / \varepsilon)\right\|_{H^{1}(\omega)}=O(\varepsilon) .
$$

Following these results, a natural attempt is to derive the next order approximation, and an estimate like

$$
\left\|u^{\varepsilon}-u^{0}(x)-\varepsilon u^{1}(x, x / \varepsilon)-\varepsilon^{2} u^{2}(x, x / \varepsilon)\right\|_{H^{1}(\omega)}=O\left(\varepsilon^{2}\right) .
$$

However, to obtain this refined approximation turns out to be difficult, and very much dependent on the geometry of $\Omega$. Before stating our results on this problem, let us describe its main difficulties and former studies.

To establish the estimate (1.8), one must first identify the average part $\bar{u}^{1}(x)$ and the oscillating term $\tilde{u}^{2}(x, y)$. Note that the choice of $\bar{u}^{1}(x)$ did not affect previous estimates (1.6), (1.7). Following Allaire and Amar [1], one needs to introduce another family of 1-periodic matrices

$$
\Upsilon^{\alpha \beta}=\Upsilon^{\alpha \beta}(y) \in M_{n}(\mathbb{R}), \quad \alpha, \beta=1, \ldots, d,
$$

satisfying

$$
-\nabla_{y} \cdot A \nabla_{y} \Upsilon^{\alpha \beta}=B^{\alpha \beta}-\int_{y} B^{\alpha \beta}, \quad \int_{y} \Upsilon^{\alpha \beta}=0
$$

where

$$
B^{\alpha \beta}:=A^{\alpha \beta}-A^{\alpha \gamma} \frac{\partial \chi^{\beta}}{\partial y_{\gamma}}-\frac{\partial}{\partial y_{\gamma}}\left(A^{\gamma \alpha} \chi^{\beta}\right) .
$$

Formal considerations yield

$$
u^{2}(x, y):=\Upsilon^{\alpha, \beta} \frac{\partial^{2} u^{0}}{\partial x_{\alpha} \partial x_{\beta}}-\chi^{\alpha} \partial_{\alpha} \bar{u}^{1} .
$$

The average term $\bar{u}^{1}=\bar{u}^{1}(x)$ formally satisfies the equation

$$
-\nabla \cdot A^{0} \nabla \bar{u}^{1}=c^{\alpha \beta \gamma} \frac{\partial^{3} u^{0}}{\partial x_{\alpha} \partial x_{\beta} \partial x_{\gamma}}, \quad c^{\alpha \beta \gamma}:=\int_{y} A^{\gamma \eta} \frac{\partial \Upsilon^{\alpha \beta}}{\partial y_{\eta}}-A^{\alpha \beta} \chi^{\gamma} .
$$

We refer to [1] for all details. Note that $u^{2}$ depends on $\bar{u}^{1}$, and has zero average with respect to $y$. In other words, we take $\bar{u}^{2}=0$. This is enough for an $O\left(\varepsilon^{2}\right)$ approximation, in the same way as taking $\bar{u}^{1}=0$ was enough to obtain an $O(\varepsilon)$ approximation.

Note also that these relations are not enough: to close system (1.11), boundary conditions on $\bar{u}^{1}$ are required. To derive the correct boundary conditions and obtain the interior estimate (1.8), one needs to understand the behavior of $u^{\varepsilon}$ near the boundary. This is emphasized in [1, Theorem 3.7], where it is shown that

$$
\left\|u^{\varepsilon}-u^{0}(x)-\varepsilon u^{1}(x, x / \varepsilon)-\varepsilon u_{b l}^{1, \varepsilon}(x)-\varepsilon^{2} u^{2}(x, x / \varepsilon)\right\|_{H^{1}(\Omega)}=O\left(\varepsilon^{3 / 2}\right),
$$


with $u_{b l}^{1, \varepsilon}(x)$ the solution of the Dirichlet problem

$$
\left\{\begin{aligned}
-\nabla \cdot A(x / \varepsilon) \nabla u_{b l}^{1, \varepsilon}=0, & x \in \Omega \subset \mathbb{R}^{d}, \\
u_{b l}^{1, \varepsilon}=-u^{1}(x, x / \varepsilon), & x \in \partial \Omega .
\end{aligned}\right.
$$

In other words, the construction of high order approximation relies on the homogenization of system (1.12). The main problem is that the homogenization of this auxiliary system is much harder than the original one. Indeed, the boundary data in (1.12) forces oscillations within a boundary layer. To understand the structure of these (not anymore periodic) oscillations and their averaged effect is essentially an open question.

Most works on that topic have been limited to convex polygons

$$
\Omega:=\bigcap_{k=1}^{N}\left\{x: n^{k} \cdot x>c^{k}\right\},
$$

bounded by $N$ hyperplanes of $\mathbb{R}^{d}$ with inward unit normal vector $n^{k}$ :

$$
K^{k}:=\left\{x: n^{k} \cdot x=c^{k}\right\}, \quad n^{k} \in S^{d-1}, c^{k} \in \mathbb{R}, 1 \leq k \leq N,
$$

More precisely, all results have been obtained under the stringent assumption that the normal vector $n^{k}$ can be taken in $\mathbb{R Q}^{d}$, that is, proportional to a vector with rational coordinates. When $d=2$, this corresponds to polygons with sides of rational slopes, and we will keep this terminology for general $d$. For instance, in [1], Allaire and Amar consider the special case

$$
\Omega=[0,1]^{d}, \quad \varepsilon_{n}=1 / n .
$$

They manage to build correctors such that a bound of type (1.8) holds when $\varepsilon=\varepsilon_{n}$. They show that the appropriate boundary conditions on $\bar{u}^{1}$ read

$$
\bar{u}^{1}=\Gamma^{k} \partial_{n} u^{0}, \quad x \in K^{k} \cap \partial \Omega, 1 \leq k \leq N,
$$

with the matrix coefficients $\Gamma^{k} \in M_{n}(\mathbb{R})$ linked to some auxiliary boundary layer systems. Numerical schemes based on these correctors are studied in [20, 18]. Let us also mention [15], where the case of layered media is considered.

We point out that this construction of accurate approximations originates in a series of papers by Vogelius and co-authors $[17,16,13]$, within the slightly different context of eigenvalue problems

$$
\left\{\begin{aligned}
-\nabla \cdot A(x / \varepsilon) \nabla u^{\varepsilon}=\lambda^{\varepsilon} u^{\varepsilon}, & x \in \Omega \subset \mathbb{R}^{d}, \\
u^{\varepsilon}=0, & x \in \partial \Omega .
\end{aligned}\right.
$$

The behavior of $\lambda^{\varepsilon}$ is investigated, notably the accumulation points of the ratio

$$
\frac{\lambda^{\varepsilon}-\lambda^{0}}{\varepsilon} \quad \text { as } \varepsilon \rightarrow 0
$$

when $\lambda^{0}$ is a simple eigenvalue of the homogenized system (1.3). The analysis is performed in the case of convex polygons with sides of rational slopes, and relies on the 
boundary layer systems later used in [1]. It is shown that the ratio does not in general have one limit but rather a continuum of accumulation points. Recast in the framework of the article [1], with $\Omega=[0,1]^{d}$, this result indicates that the constant matrices $\Gamma^{k}$ in (1.13) depend on the subsequence $\varepsilon_{n}$, so that the corrector $\bar{u}^{1}$ in the approximation (1.8) also depends on the subsequence $\varepsilon_{n}$ (which is $\varepsilon_{n}=1 / n$ in [1]). Crudely, one can then say that for convex polygons with sides of rational slopes, estimate (1.8) does not hold uniformly in $\varepsilon$.

The aim of this paper is to consider general convex polygonal domains $\Omega$, that is, without the assumption of rational slopes. We will show that "generically", there exists an $O\left(\varepsilon^{2}\right)$ two-scale approximation of $u^{\varepsilon}$ inside $\Omega$.

Our main assumption will be a diophantine condition on the normals $n:=n^{k}, k=$ $1, \ldots, N$ :

(A) There are $c, l>0$ such that for all $\xi \in \mathbb{Z}^{d} \backslash\{0\},|n \times \xi| \geq c|\xi|^{-l}$,

where $n \times \xi:=n_{2} \xi_{1}-n_{1} \xi_{2}$ when $d=2$, and $n \times \xi$ is the usual cross product when $d=3$. If $d=2$, one can replace the cross product in assumption (A) by a scalar product, namely $|n \cdot \xi| \geq c|\xi|^{-l}$. If $d=3$, then assumption (A) is equivalent to the fact that any two components of $n$, say $\left(n_{1}, n_{2}\right)$, satisfy: for all $\xi \in \mathbb{Z}^{2} \backslash\{0\},\left|n_{1} \xi_{1}+n_{2} \xi_{2}\right| \geq c|\xi|^{-l}$. We emphasize that this condition is generic, in the sense that it is satisfied for almost every $n^{1}, \ldots, n^{N}$. This is a direct consequence of the following classical result (see [7]): For almost every vector $v \in \mathbb{R}^{d}$, and all $\delta>0$, there exists $c>0$ such that

$$
|v \cdot \xi| \geq c|\xi|^{-d-\delta}, \quad \forall \xi \in \mathbb{Z}^{d} \backslash\{0\}
$$

Besides this small divisor assumption, we will need technical assumptions on $u^{0}, u^{1}$, due to possible loss of regularity near the edges and vertices of $\Omega$. Namely, we will assume that

(A0) The solution $u^{0}$ of (1.3) belongs to $H^{3}(\Omega) \cap C^{2}(\bar{\Omega})$.

(A1) The solution $\bar{u}^{1}$ of (1.11)-(1.13) with $\Gamma^{k}$ defined in (3.2) belongs to $H^{2}(\Omega) \cap C^{1}(\bar{\Omega})$.

The relevance of hypothesis (A0), the well-posedness of (1.11)-(1.13), and the relevance of hypothesis (A1) will be discussed extensively in Section 3.

We can state our main result:

Theorem 1. Let $\Omega=\bigcap_{k=1}^{N}\left\{x: n^{k} \cdot x>c^{k}\right\}$ be a convex polygonal domain. Suppose that for all $k$, the normal vector $n=n^{k}$ satisfies the diophantine condition $(\mathbf{A})$, and that the regularity conditions (A0) and (A1) hold. Then, for any open subset $\omega \Subset \Omega$,

$$
\left\|u^{\varepsilon}-u^{0}(x)-\varepsilon u^{1}(x, x / \varepsilon)-\varepsilon^{2} u^{2}(x, x / \varepsilon)\right\|_{H^{1}(\omega)}=O\left(\varepsilon^{2}\right),
$$

with $u^{0}, \bar{u}^{1}$ as in (A0) and (A1), and $u^{1}, u^{2}$ as in (1.5) and (1.10).

The technical constraints (A0)-(A1) being set aside, this shows that for generic polygonal domains, there exists an $\varepsilon^{2}$ two-scale approximation of $u^{\varepsilon}$. Note that the higher order correction in (1.8) is independent of the subsequence in $\varepsilon$. In that respect, the case of 
rational slopes is peculiar. In this case, as can be deduced from [1,13] in the periodic case, the higher order correction may depend on the sequence.

The main part of the proof of Theorem 1 is the treatment of the boundary layer. In previous studies, the rational slopes allowed to get periodicity in the tangential variable. In the case of general irrational slopes, only a quasiperiodicity property is available, making the construction of boundary layer correctors more intricate. The construction is performed in Section 2. The derivation of $u^{1}, u^{2}$, and the proof of estimate (1.8) follows in Section 3. As we will see from the proof, we have a more precise version of Theorem 1 (see Corollary 8).

\section{Homogenization of the boundary layer}

\subsection{Formal expansion}

As emphasized in the introduction, the search for high order approximations reduces to the understanding of the Dirichlet problem (1.12). Formally, one expects $u_{b l}^{1, \varepsilon}$ to be localized in the vicinity of the hyperplanes of $\Omega$ :

$$
u_{b l}^{1, \varepsilon}(x)=\sum_{k=1}^{N} u_{b l}^{1, \varepsilon, k}(x)
$$

where $u_{b l}^{1, \varepsilon, k}(x)$ describes a boundary layer near $K^{k}$. Note that by convexity, $\Omega$ lies on one side of $K^{k}$, for all $1 \leq k \leq N$. Hence,

$$
\Omega \subset\left\{x: n^{k} \cdot x-c^{k}>0\right\} .
$$

We look for an approximation of the type

$$
u_{b l}^{1, \varepsilon, k} \approx v_{b l}^{k}(x, x / \varepsilon),
$$

where $v_{b l}^{k}=v_{b l}^{k}(x, y) \in \mathbb{R}^{n}$ is defined for $x \in \Omega$, and $y$ is in the half-space

$$
\Omega^{\varepsilon, k}=\left\{y: n^{k} \cdot y-c^{k} / \varepsilon>0\right\} .
$$

Plugging this approximation in (1.12) yields

$$
\left\{\begin{aligned}
-\nabla_{y} \cdot A(y) \nabla_{y} v_{b l}^{k}=0, & y \in \Omega^{\varepsilon, k} \\
v_{b l}^{k}=-u_{1}(x, y), & y \in \partial \Omega^{\varepsilon, k} .
\end{aligned}\right.
$$

Note that the variable $x$ is only a parameter in this system. Let $M^{k}$ be an orthogonal matrix that maps the canonical vector $e_{d}=(0, \ldots, 0,1)$ to the normal vector $n^{k}$. By the change of variable $y=M^{k} z$, system (2.1) becomes

$$
\left\{\begin{array}{rr}
-\nabla_{z} \cdot B^{k}\left(M^{k} z\right) \nabla_{z} v^{k}=0, & z_{d}>c^{k} / \varepsilon \\
v^{k}=-u_{1}\left(x, M^{k} z\right), & z_{d}=c^{k} / \varepsilon
\end{array}\right.
$$


with unknown $v^{k}(x, z)=v_{b l}^{k}\left(x, M^{k} z\right)$. Denoting by $A_{i j}^{\alpha \beta}$, resp. $B_{i j}^{k, \alpha \beta}, 1 \leq i, j \leq n$, the coefficients of $A^{\alpha \beta}$, resp. $B^{k, \alpha \beta}$, we recall the relation

$$
\forall i, j, \quad B_{i j}^{k}=M^{k} A_{i j}\left(M^{k}\right)^{t}
$$

which is a product of matrices in $M_{d}(\mathbb{R})$. We also denote by $z=\left(z^{\prime}, z_{d}\right)$ the tangential and normal component of $z$. We stress that $v_{b l}^{k}$ and $v^{k}$ still depend on $\varepsilon$, through the $c^{k} / \varepsilon$ term. As will be clear from the developments below, this dependence is harmless, so that we omit it in notation.

The proof of Theorem 1 relies mostly on the analysis of system (2.2). In the case of polygons with sides of rational slopes, for which $n_{k}$ belongs to $\mathbb{R} \mathbb{Q}^{d}$, one can choose a matrix $M^{k}$ with columns that are also in $\mathbb{R Q}^{d}$, so that system (2.2) has coefficients that are still periodic in $z^{\prime}$. Working in spaces of functions periodic in $z^{\prime}$, one easily obtains existence and uniqueness of a variational solution. Moreover, using a lemma of Tartar, one can show the convergence of this solution towards a constant, as $z_{d}$ goes to infinity, exponentially fast. We refer to [1] for all details. The basic ingredient used in the study of this rational case is the Poincaré inequality

$$
\int_{\mathbb{T}^{d-1}}|\tilde{\varphi}|^{2} d z^{\prime} \leq C \int_{\mathbb{T}^{d-1}}|\nabla \tilde{\varphi}|^{2} d z^{\prime}
$$

for $L$-periodic functions $\tilde{\varphi}$ with zero average.

These properties fail to be true for general polygons: the coefficients are not anymore periodic, but quasiperiodic. We refer to [11] for a description of quasiperiodic and almost periodic functions. Quasiperiodicity does not allow one to restrict the tangential variable to a bounded domain, and Poincaré's inequality is not anymore valid. As detailed in the next subsection, we will still be able to deal with system (2.2), under the generic diophantine assumption (A).

\subsection{Boundary layer system}

Directly inspired by (2.2), we introduce the following system:

$$
\left\{\begin{aligned}
-\nabla_{z} \cdot B(M z) \nabla_{z} v=0, & z_{d}>a, \\
v(z)=v_{0}(M z), & z_{d}=a,
\end{aligned}\right.
$$

where $B$ shares the same properties as the original matrix $A, v_{0}$ is a smooth 1-periodic function and $M$ is a $d \times d$ orthogonal matrix. We wish to show the well-posedness of this system. Moreover, as in the case of rational slopes, we expect the solution to converge towards a constant vector as $z_{d}$ goes to infinity. Let $N \in M_{d, d-1}(\mathbb{R})$ be defined by

$$
N z^{\prime}=M\left(z^{\prime}, 0\right) \text {. }
$$

The structure of (2.3) suggests looking for a solution of the type

$$
v(z)=V\left(N z^{\prime}, z_{d}\right), \quad V(\theta, t) 1 \text {-periodic in } \theta \in \mathbb{R}^{d} .
$$


Accordingly, we define

$$
\mathcal{B}(\theta, t)=B(\theta+M(0, t)), \quad V_{0}(\theta, t)=v_{0}(\theta+M(0, t)) .
$$

This leads to the following system, for $\theta \in \mathbb{T}^{d}$ and $t>a$ :

$$
\left\{\begin{array}{r}
-\left(\begin{array}{c}
N^{t} \nabla_{\theta} \\
\partial_{t}
\end{array}\right) \cdot \mathcal{B}(\theta, t)\left(\begin{array}{c}
N^{t} \nabla_{\theta} \\
\partial_{t}
\end{array}\right) V=0, \quad t>a, \\
V(\theta, t=a)=V_{0}(\theta, t=a), \quad t=a .
\end{array}\right.
$$

As this new formulation reveals, the solvability of (2.5) is unclear. The problem is the lack of coerciveness of the new operator with respect to $\theta$. For instance, we do not have in general

$$
\int_{\mathbb{T}^{d}}\left|N^{t} \nabla_{\theta} \phi\right|^{2} d \theta \geq c \int_{\mathbb{T}^{d}}\left|\nabla_{\theta} \phi\right|^{2} d \theta .
$$

This can be understood easily in the two-dimensional case: if $M$ is a rotation matrix

$$
M=\left(\begin{array}{cc}
\cos \alpha & -\sin \alpha \\
\sin \alpha & \cos \alpha
\end{array}\right), \text { then } N=\left(\begin{array}{c}
\cos \alpha \\
\sin \alpha
\end{array}\right),
$$

and inequality (2.6) would give (using the Plancherel identity): for all $\xi_{1}, \xi_{2} \in \mathbb{Z}^{2}$,

$$
\left(\xi_{1} \cos \alpha+\xi_{2} \sin \alpha\right)^{2} \geq c\left(\left|\xi_{1}\right|^{2}+\left|\xi_{2}\right|^{2}\right),
$$

which is never satisfied uniformly for large $\xi_{1}, \xi_{2}$. The well-posedness issue is considered in the next subsection.

Another issue to be considered after well-posedness is the asymptotic behavior of $V$ as $t \rightarrow+\infty$. Arguments in [1] for the periodic setting do not adapt to our quasiperiodic setting. To overcome this difficulty, we will make a crucial use of the small divisor assumption (A). Note that a straightforward reformulation is

(A) There exist $c, l>0$ such that for all $\xi \in \mathbb{Z}^{d} \backslash\{0\},\left|N^{t} \xi\right| \geq c|\xi|^{-l}$.

It will be used in this form to show convergence to a constant field at infinity.

\subsection{Well-posedness}

We have the following well-posedness result for system (2.5):

Proposition 2. There exists a unique smooth solution $V$ of (2.5) such that

$$
\int_{\mathbb{T}^{d}} \int_{a}^{+\infty}\left(\left|N^{t} \nabla_{\theta} \partial_{\theta}^{\gamma} V\right|^{2}+\left|\partial_{t}^{l} \partial_{\theta}^{\gamma} V\right|^{2}\right) d t d \theta<+\infty
$$

for $l \geq 1$ and $\gamma \in \mathbb{N}^{d}$ and where we denote $\partial_{\theta}^{\gamma}=\partial_{\theta_{1}}^{\gamma_{1}} \ldots \partial_{\theta_{d}}^{\gamma_{d}}$. As a consequence, $v(z)=$ $V\left(N z^{\prime}, z_{d}\right)$ is a smooth solution of (2.3).

The proof of the proposition relies on the following simple estimate. 
Lemma 3. If $Y(\theta, t)$ is a smooth function solving

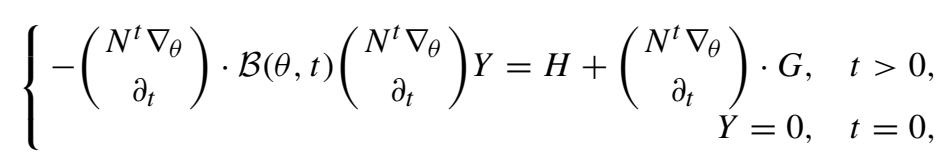

where t $H, G \in L^{2}\left(\mathbb{T}^{d} \times \mathbb{R}_{+}\right)$, then

$$
\int_{\mathbb{T}^{d}} \int_{0}^{+\infty}\left(\left|N^{t} \nabla_{\theta} Y\right|^{2}+\left|\partial_{t} Y\right|^{2}\right) d t d \theta \leq C \int_{\mathbb{T}^{d}} \int_{0}^{+\infty}\left(|t H|^{2}+|G|^{2}\right) d t d \theta .
$$

Proof of the lemma. Multiplying by $Y$ and integrating over $\mathbb{T}^{d} \times \mathbb{R}^{+}$, we obtain

$$
\begin{aligned}
\int_{\mathbb{T}^{d}} \int_{0}^{+\infty}\left(\left|N^{t} \nabla_{\theta} Y\right|^{2}+\left|\partial_{t} Y\right|^{2}\right) d t d \theta \leq & \int_{\mathbb{T}^{d}} \int_{0}^{+\infty}(t H) \cdot \frac{Y}{t} d t d \theta \\
& +\int_{\mathbb{T}^{d}} \int_{0}^{+\infty} G \cdot\left(\begin{array}{c}
N^{t} \\
\partial_{\theta}
\end{array}\right) Y d t d \theta .
\end{aligned}
$$

By Hardy's inequality,

$$
\left\|\frac{Y}{t}\right\|_{L^{2}\left(\mathbb{T}^{d} \times \mathbb{R}^{+}\right)} \leq C\left\|\partial_{t} Y\right\|_{L^{2}\left(\mathbb{T}^{d} \times \mathbb{R}^{+}\right)} \leq C\left\|\left(\begin{array}{c}
N^{t} \nabla_{\theta} \\
\partial_{t}
\end{array}\right) Y\right\|_{L^{2}\left(\mathbb{T}^{d} \times \mathbb{R}^{+}\right)} .
$$

Using this bound and the Cauchy-Schwarz inequality in the previous inequality yields the result.

Proof of the proposition. Without loss of generality, one can assume $a=0$. Let $\delta(t)$ be a smooth truncation function satisfying $\delta=1$ on $[0,1 / 2]$ and $\delta=0$ outside $[0,1]$. Introducing

$$
Y=V-\delta(t) V_{0},
$$

the problem reduces to the well-posedness of

$$
\left\{\begin{aligned}
-\left(\begin{array}{c}
N^{t} \nabla_{\theta} \\
\partial_{t}
\end{array}\right) \cdot \mathcal{B}(\theta, t)\left(\begin{array}{c}
N^{t} \nabla_{\theta} \\
\partial_{t}
\end{array}\right) Y=F, & t>0 \\
Y=0, & t=0
\end{aligned}\right.
$$

where $F$ is smooth, periodic in $\theta$, and has support in $t \leq 1$.

A priori estimates. Suppose $Y$ is a smooth solution of system (2.9). Using (2.8) with $H=F$ and $G=0$ yields the $L^{2}$ estimate

$$
\int_{\mathbb{T}^{d}} \int_{0}^{+\infty}\left(\left|N^{t} \nabla_{\theta} Y\right|^{2}+\left|\partial_{t} Y\right|^{2}\right) d t d \theta \leq C \int_{\mathbb{T}^{d}} \int_{0}^{+\infty}|F|^{2} d t d \theta
$$

The same type of estimate extends easily to tangential derivatives. Namely, for $|\gamma| \geq 0$,

$$
\iint\left(\left|N^{t} \nabla_{\theta} \partial_{\theta}^{\gamma} Y\right|^{2}+\left|\partial_{t} \partial_{\theta}^{\gamma} Y\right|^{2}\right) d t d \theta \leq C(\gamma) \sum_{|\beta| \leq|\gamma|} \iint\left|\partial_{\theta}^{\beta} F\right|^{2} d t d \theta .
$$


Indeed, for $|\gamma|=1$, we differentiate (2.9) with respect to $\partial_{\theta_{\alpha}}$ for some $1 \leq \alpha \leq d$ and then apply Lemma 3 with $H=\partial_{\theta_{\alpha}} F$ and $G=\partial_{\theta_{\alpha}} \mathcal{B}(\theta, t)\left(\begin{array}{c}N^{t} \nabla_{\theta} \\ \partial_{t}\end{array}\right) Y$. The general case is obtained by induction on the number of derivatives.

Then standard elliptic arguments provide additional regularity with respect to $t$. We first notice that equation (2.9) can be written

$$
\mathcal{B}_{d, d} \partial_{t}^{2} Y=G \quad \text { with } G \in L_{t}^{2}\left(H^{s}\left(\mathbb{T}^{d}\right)\right), \forall s \in \mathbb{N},
$$

where we used (2.11) to estimate $G$ and where $\mathcal{B}_{d, d}^{i j}=M_{d \alpha} A_{\alpha \beta}^{i j}{ }^{t} M_{\beta d}$ satisfies the coercivity condition $\left|\mathcal{B}_{d, d} \xi \cdot \xi\right| \geq \lambda|\xi|$ for $\xi \in \mathbb{R}^{n}$.

Inverting $\mathcal{B}_{d, d}$, we deduce the same regularity for $\partial_{t}^{2} Y$, which implies that $\left.\partial_{t} Y\right|_{t=0}$ belongs to $H^{s}\left(\mathbb{T}^{d}\right)$ for all $s$. Hence, we may differentiate the equation in $t$, recover a homogeneous Dirichlet condition by a change of unknown, and apply the previous arguments. Reasoning recursively, we obtain easily: for all $\gamma \in \mathbb{N}^{d}$, and all $k \geq 1$,

$$
\int_{\mathbb{R}^{+}}\left(\left\|N^{t} \nabla_{\theta} \partial_{\theta}^{\gamma} Y\right\|_{H^{s}\left(\mathbb{T}^{d}\right)}^{2}+\left\|\partial_{t}^{k} Y\right\|_{H^{s}\left(\mathbb{T}^{d}\right)}^{2}\right) \leq C(F, s, k)<+\infty .
$$

We point out here that we lack an estimate for $Y$ itself, that is, without any derivative.

Well-posedness. The existence of solutions that satisfy the previous energy estimate can be obtained from standard elliptic regularization of the system. One can for instance consider the approximate problems

$$
\left\{\begin{array}{r}
-\delta \Delta_{\theta} V-\left(\begin{array}{c}
N^{t} \nabla_{\theta} \\
\partial_{t}
\end{array}\right) \cdot \mathcal{B}(\theta, t)\left(\begin{array}{c}
N^{t} \nabla_{\theta} \\
\partial_{t}
\end{array}\right) V=0, \quad t>a \\
V(\theta, t=a)=V_{0}(\theta, t=a), \quad t=a
\end{array}\right.
$$

for a small parameter $\delta>0$. As the system is strongly elliptic for each $\delta$, one can easily show existence and uniqueness of a smooth solution $V_{\delta}$ that satisfies all previous estimates uniformly with respect to $\delta$. As $\delta \rightarrow 0$, one easily gets a smooth solution $V$ of (2.5). Uniqueness follows from the basic estimate (2.10).

\subsection{Behavior at infinity}

The next step in the study of the boundary layer is to understand the behavior of $V$ as $t$ goes to infinity. In this subsection, we will use the assumption (A) to prove the existence of a limit when $t$ goes to infinity for $V$. First, assumption (A) ensures the following inequality:

$$
\int_{\mathbb{T}^{d}}\left|N^{t} \nabla_{\theta} \tilde{\varphi}\right|^{2} \geq c\|\tilde{\varphi}\|_{H^{-l}\left(\mathbb{T}^{d}\right)}^{2}
$$

for smooth enough $\tilde{\varphi}=\tilde{\varphi}(\theta)$ with zero average. Combining (2.14) with (2.11), we deduce that for any $s \in \mathbb{N}$,

$$
\int_{a}^{+\infty}\left(\|\tilde{V}\|_{H^{s}\left(\mathbb{T}^{d}\right)}^{2}+\left\|\partial_{t}^{k} V\right\|_{H^{s}\left(\mathbb{T}^{d}\right)}^{2}\right) \leq C(F, s, k)<+\infty,
$$


where we decompose

$$
V(\theta, t)=\tilde{V}(\theta, t)+\bar{V}(t), \quad \int_{\mathbb{T}^{d}} \tilde{V} d \theta=0 .
$$

This implies that for all $\gamma \in \mathbb{N}^{d}$ and $k \in \mathbb{N}$, we have, uniformly in $\theta$

$$
\partial_{\theta}^{\gamma} \partial_{t}^{k} \tilde{V} \rightarrow 0, \quad \partial_{\theta}^{\gamma} \partial_{t}^{k+1} V \rightarrow 0, \quad t \rightarrow+\infty .
$$

However, the behavior of the average $\bar{V}$ and the speed of convergence are not specified. This is the purpose of the next proposition.

Proposition 4. There exists a constant vector $v^{a} \in \mathbb{R}^{n}$ such that

$$
\lim _{t \rightarrow+\infty} V=v^{a}
$$

More precisely,

$$
\lim _{t \rightarrow+\infty}\left|t^{m} \partial_{\theta}^{\gamma} \partial_{t}^{k}\left(V-v^{a}\right)\right|=0
$$

for all $m \in \mathbb{N}, \gamma \in \mathbb{N}^{d}, k \in \mathbb{N}$, uniformly in $\theta$.

Note that the solution $V$ of (2.5) depends on $a$ (and also on $B, M, V_{0}$ ), a fact that we have omitted so far in our notation. Here, we only keep track of this dependence in the limit $v^{a}$, as it will be of interest to us later on.

Proof. To prove Proposition 4, we establish an integro-differential inequality for

$$
f(T):=\int_{\mathbb{T}^{d}} \int_{T}^{+\infty}\left(\left|N^{t} \nabla_{\theta} V\right|^{2}+\left|\partial_{t} V\right|^{2}\right) d t d \theta .
$$

Let $T>a$, and for $t \geq T$, define

$$
W:=V-\int_{\mathbb{T}^{d}} V(\theta, T) d \theta .
$$

For $t \geq T, W$ satisfies

$$
-\left(\begin{array}{c}
N^{t} \nabla_{\theta} \\
\partial_{t}
\end{array}\right) \cdot \mathcal{B}(\theta, t)\left(\begin{array}{c}
N^{t} \nabla_{\theta} \\
\partial_{t}
\end{array}\right) W=0
$$

Multiplying by $W$ and integrating over $\theta \in \mathbb{T}^{d}, t \geq T$, we get

$$
\begin{gathered}
\int_{\mathbb{T}^{d}} \int_{T}^{+\infty}\left(\left|N^{t} \nabla_{\theta} W\right|^{2}+\left|\partial_{t} W\right|^{2}\right)=-\int_{\mathbb{T}^{d}}\left[\left(\begin{array}{c}
0_{d-1} \\
1
\end{array}\right) \cdot \mathcal{B}\left(\begin{array}{c}
N^{t} \nabla_{\theta} \\
\partial_{t}
\end{array}\right) W\right] W(\theta, T) d \theta \\
\leq C\left(\int_{\mathbb{T}^{d}}\left(\left|N^{t} \nabla_{\theta} W\right|^{2}+\left|\partial_{t} W\right|^{2}\right)(\theta, T) d \theta\right)^{1 / 2}\left(\int_{\mathbb{T}^{d}}|W(\theta, T)|^{2} d \theta\right)^{1 / 2} .
\end{gathered}
$$


As $\nabla_{\theta} W=\nabla_{\theta} V, \partial_{t} W=\partial_{t} V$, and $W(\theta, T)=\tilde{V}(\theta, T)$, this last inequality reads

$$
f(T) \leq C\left(-f^{\prime}(T)\right)^{1 / 2}\left(\int_{\mathbb{T}^{d}}|\tilde{V}(\theta, T)|^{2} d \theta\right)^{1 / 2} .
$$

Now, by assumption (A), for all $1<p<+\infty$, and all smooth enough $\tilde{\varphi}$ with zero average, we have

$$
\int_{\mathbb{T}^{d}}|\tilde{\varphi}|^{2} d \theta \leq C\left(\int_{\mathbb{T}^{d}}\left|N^{t} \nabla_{\theta} \tilde{\varphi}\right|^{2}\right)^{1 / p}\left(\|\tilde{\varphi}\|_{H^{l /(p-1)}\left(\mathbb{T}^{d}\right)}\right)^{2-2 / p},
$$

where the index $l$ is the same as in (A). This inequality is a straightforward consequence of the Plancherel formula and the Hölder inequality (together with the small divisor assumption). Applying this to $\tilde{V}(\theta, T)$, we obtain

$$
\int_{\mathbb{T}^{d}}|\tilde{V}(\theta, T)|^{2} d \theta \leq\left(-f^{\prime}(T)\right)^{1 / p}\left(\|\tilde{V}(\cdot, T)\|_{H^{l /(p-1)}\left(\mathbb{T}^{d}\right)}\right)^{2-2 / p} \leq C\left(-f^{\prime}(T)\right)^{1 / p}
$$

bounding the last term thanks to (2.15). This yields the integro-differential inequality

$$
f(T) \leq C(p)\left(-f^{\prime}(T)\right)^{\frac{p+1}{2 p}}
$$

for any $1<p<+\infty$. This leads in turn to

$$
f(T) \leq C^{\prime}(p) T^{\frac{p+1}{1-p}} .
$$

It shows that $f(T)$ decays faster than any power of $T$ as $T$ goes to infinity.

By differentiation of $(2.5)_{1}$ and similar estimates, one shows by induction on $|\alpha|+k$ that

$$
f_{\gamma, k}(T):=\int_{\mathbb{T}^{d}} \int_{T}^{+\infty}\left(\left|N^{t} \nabla_{\theta} \partial_{\theta}^{\gamma} \partial_{t}^{k} V\right|^{2}+\left|\partial_{t} \partial_{\theta}^{\gamma} \partial_{t}^{k} V\right|^{2}\right) d t d \theta
$$

decays faster than any power of $T$, for any $\gamma, k$. More precisely, assuming that such decay holds for all $f_{\beta, l}$ with $|\beta|+l<s$, the energy estimate (2.16) is easily replaced by

$$
f_{\gamma, k}(T) \leq C(p, n)\left(\left(-f_{\gamma, k}^{\prime}(T)\right)^{\frac{p+1}{2 p}}+T^{-n}\right), \quad \forall n, p>1, \forall \gamma, k \text { with }|\gamma|+k=s .
$$

Hence, one gets

$$
f_{\gamma, k}(T)+T^{\frac{p+1}{1-p}} \leq C\left(\left(-f_{\gamma, k}^{\prime}(T)\right)^{\frac{p+1}{2 p}}+T^{\frac{p+1}{1-p}}\right) \leq C^{\prime}\left(-f_{\gamma, k}^{\prime}(T)+T^{\frac{2 p}{1-p}}\right)^{\frac{p+1}{2 p}},
$$

that is,

$$
g_{\gamma, k}(T) \leq C^{\prime \prime}\left(-g_{\gamma, k}^{\prime}(T)\right)^{\frac{p+1}{2 p}}, \quad g_{\gamma, k}(T):=f_{\gamma, k}(T)+T^{\frac{p+1}{1-p}},
$$

and one can conclude as above.

Using again (2.14) and Sobolev imbedding, we deduce that

$$
\lim _{t \rightarrow+\infty}\left|t^{m} \partial_{\theta}^{\gamma} \partial_{t}^{k} \tilde{V}\right|=0, \quad \lim _{t \rightarrow+\infty}\left|t^{m} \partial_{\theta}^{\gamma} \partial_{t}^{k+1} V\right|=0,
$$

for all $m \in \mathbb{N}, \gamma \in \mathbb{N}^{d}, k \in \mathbb{N}$, uniformly in $\theta$. 
It remains to show the convergence of the average $\bar{V}=\bar{V}(t)$. We write

$$
|\bar{V}(t+h)-\bar{V}(t)| \leq \int_{t}^{t+h}\left|\frac{d}{d t} \bar{V}\right| \leq C(p) \int_{t}^{t+h}(1+s)^{-p} d s
$$

for all $p$. This shows that $\bar{V}(t)$ is a Cauchy function, hence convergent to a constant vector $v^{a}$ as $t$ goes to infinity. Moreover, the rate of convergence is faster than any power function of $t$.

Back to the original system (2.3), previous results provide a unique smooth solution $v=v(z)$ that converges to a constant $v^{a}$ as $z_{d} \rightarrow+\infty$. Looking closer at Proposition 4 and its proof, we have: for all $m \in \mathbb{N}, \gamma \in \mathbb{N}^{d-1}, k \in \mathbb{N}$,

$$
\lim _{z_{d}-a \rightarrow+\infty}\left|\left(z_{d}-a\right)^{m} \partial_{z^{\prime}}^{\gamma} \partial_{z_{d}}^{k}\left(v-v^{a}\right)\right|=0
$$

locally uniformly in $z^{\prime}$, and uniformly in $a$. We end this section with a crucial property of the constant vector $v^{a}$.

Proposition 5. Let $M$ be the matrix given in (2.3), and $e_{d}=(0, \ldots, 0,1)$ the $d$-th canonical vector. If $M e_{d} \notin \mathbb{R Q}^{d}$, then $v^{a}$ is independent of $a$.

Note that in the case $M=M^{k}$ (cf. (2.2)), $M e_{d}=n^{k}$ is a normal vector at $\partial \Omega \cap K^{k}$.

Proof. We start from the following lemma:

Lemma 6. $v^{a}$ depends continuously on a.

Proof of the lemma. Let $a$ and $a^{\prime}$ be two real values, and $V, V^{\prime}$ the corresponding solutions of (2.5). We denote $\delta=a^{\prime}-a$. We introduce

$$
V_{\delta}^{\prime}(\theta, t)=V^{\prime}(\theta, t+\delta), \quad \theta \in \mathbb{T}^{d}, t>a .
$$

We have

$$
\left|V_{\delta}^{\prime}(\theta, t)-V^{\prime}(\theta, t)\right| \leq|\delta|\left\|\partial_{t} V^{\prime}\right\|_{L^{\infty}} \leq C|\delta| .
$$

Now, $V$ and $V_{\delta}^{\prime}$ are defined on the same domain, and $W=V-V_{\delta}^{\prime}$ satisfies

$$
\left\{\begin{array}{r}
-\left(\begin{array}{c}
N^{t} \nabla_{\theta} \\
\partial_{t}
\end{array}\right) \cdot \mathcal{B}(\theta, t)\left(\begin{array}{c}
N^{t} \nabla_{\theta} \\
\partial_{t}
\end{array}\right) W=F, \quad t>a, \\
W=W_{0}, \quad t=a,
\end{array}\right.
$$

where

$$
F:=\left(\begin{array}{c}
N^{t} \nabla_{\theta} \\
\partial_{t}
\end{array}\right) \cdot(\mathcal{B}(\theta, t)-\mathcal{B}(\theta, t+\delta))\left(\begin{array}{c}
N^{t} \nabla_{\theta} \\
\partial_{t}
\end{array}\right) V_{\delta}^{\prime}, \quad W_{0}:=V_{0}(\theta, a)-V_{0}(\theta, a+\delta) .
$$

Note that these source terms satisfy

$$
\left|\partial_{\theta}^{\alpha} \partial_{t}^{k} F\right|+\left|\partial_{\theta}^{\alpha} \partial_{t}^{k} W_{0}\right| \leq C_{\alpha, k}|\delta|, \quad \forall \alpha, k
$$


Moreover, by Proposition 4, $F$ and its derivatives converge to zero uniformly in $\theta$, faster than any power of $t$. With this decay property, it is straightforward to adapt the energy estimates performed in the proof of Propositions 2 and 4. As a consequence, using again assumption (A), we deduce that $W$ satisfies $\|W\|_{L^{\infty}} \leq C|\delta|$, which reads

$$
\left|V(\theta, t)-V_{\delta}^{\prime}(\theta, t)\right| \leq C|\delta|
$$

uniformly in $\theta, t$. Combining (2.18), (2.20) we deduce that as $t$ goes to infinity,

$$
\left|v^{a}-v^{a^{\prime}}\right| \leq C\left|a-a^{\prime}\right|
$$

which proves the lemma.

We can now end the proof of Proposition 5. Let $\xi \in \mathbb{Z}^{d}$. If $v$ satisfies system (2.3), then $v_{\xi}(z)=v\left(z+(M)^{t} \xi\right)$ satisfies

$$
\left\{\begin{array}{rr}
-\nabla_{z} \cdot B(M z) \nabla_{z} v_{\xi}=0, & z_{d}>a-\xi \cdot M e_{d}, \\
v_{\xi}(z)=v_{0}(M z), & z_{d}=a-\xi \cdot M e_{d} .
\end{array}\right.
$$

This is deduced easily from the periodicity of $B$ and $v_{0}$ and the property $(M)^{t}=M^{-1}$. Hence, the constant at infinity satisfies

$$
v^{a}=v^{a-\xi \cdot M e_{d}}
$$

If $M e_{d} \notin \alpha \mathbb{Q}$, for any $\alpha \in \mathbb{R}$, then the set $\left\{\xi \cdot M e_{d}: k \in \mathbb{Z}^{d}\right\}$ is dense in $\mathbb{R}$, and by continuity of $v^{a}$ with respect to $a$, the result follows.

\section{High order approximation}

Thanks to the boundary layer analysis of the previous section, we shall prove Theorem 1 . From now on, we consider a convex polygonal domain $\Omega=\bigcap_{k=1}^{N}\left\{x: n^{k} \cdot x>c^{k}\right\}$ with inward normal vector $n=n^{k}$ satisfying (A) for all $k$.

\subsection{Choice of $u^{1}$ and $u^{2}$. Discussion of the assumptions (AO) and (A1)}

The first step of the proof is to derive the fields $u^{1}$ and $u^{2}$ for which (1.8) should hold. As described in the introduction, the starting point of this derivation is a formal two-scale expansion of the solution

$$
u^{\varepsilon} \approx u^{0}(x)+\varepsilon u^{1}(x, x / \varepsilon)+\varepsilon^{2} u^{2}(x, x / \varepsilon)+\cdots
$$

whose formal computation is detailed in [1]. The leading term $u^{0}$ satisfies the homogenized problem (1.3). The next order term $u^{1}$ satisfies (1.5)-(1.11). Finally, the second order term $u^{2}$ is given by (1.10). 
Of course, system (1.11) is not enough to determine $\bar{u}^{1}$, as boundary conditions must be prescribed at $\partial \Omega$. These conditions should account for boundary layer phenomena. More precisely, we expect an asymptotics of the type

$$
u^{\varepsilon} \approx u^{0}(x)+\varepsilon u^{1}(x, x / \varepsilon)+\varepsilon u_{b l}^{1, \varepsilon}(x)+\cdots
$$

where $u_{b l}^{1, \varepsilon}$ satisfies the Dirichlet problem (1.12). Following the formal considerations of Section 2 , we want to approximate this last term by

$$
u_{b l}^{1, \varepsilon}(x) \approx \sum_{k=1}^{N} v_{b l}^{k}(x, x / \varepsilon)
$$

where the boundary layer correctors $v_{b l}^{k}$ satisfy systems (2.1).

Broadly, the results of the previous section show that there exists some $v^{k, \infty}(x)$ such that

$$
v_{b l}^{k}(x, y) \rightarrow v^{k, \infty}(x) \quad \text { as } y \cdot n^{k}-c^{k} / \varepsilon \rightarrow+\infty,
$$

uniformly with respect to $x$ and $\varepsilon$. Moreover, the rate of convergence is faster than any negative power of $\left|y \cdot n^{k}-c^{k} / \varepsilon\right|$. See (2.17). The idea is to choose $\bar{u}^{1}$ at $\partial \Omega$ so that $v^{k, \infty}=0$ for all $k$. In this way, the boundary layer term should be negligible in each compact subset of $\Omega$, allowing for an estimate like (1.8). To be more specific, let $v$ be the solution of (2.3) provided by Proposition 2, under assumption (A). From Propositions 4 and 5 ,

$$
v(z) \rightarrow v^{\infty}=v^{\infty}\left[B, M, v_{0}\right] \quad \text { as } z_{d} \rightarrow+\infty, \quad \text { uniformly in } z^{\prime} .
$$

Back to systems (2.1)-(2.2), we introduce for all $1 \leq \alpha \leq d$, and all $1 \leq k \leq N$, the matrix $G^{k, \alpha} \in M^{n}(\mathbb{R})$ whose $j$-th column is defined by

$$
\left(G_{i j}^{k, \alpha}\right)_{1 \leq i \leq n}:=-v^{\infty}\left[B^{k}, M^{k},\left(\chi_{i j}^{\alpha}\right)_{1 \leq i \leq n}\right], \quad \forall 1 \leq j \leq n .
$$

Finally, we set

$$
\bar{u}^{1}=G^{k, \alpha} \frac{\partial u^{0}}{\partial x_{\alpha}}(x), \quad x \in \partial \Omega \cap K^{k}, 1 \leq k \leq N .
$$

As $u^{0}$ is zero at the boundary $\partial \Omega$, this boundary condition is the same as the Robin type condition (1.13), setting

$$
\Gamma_{i j}^{k}:=\sum_{\alpha=1}^{d} G_{i j}^{k, \alpha} n_{\alpha}^{k}, \quad n^{k}=\left(n_{\alpha}^{k}\right)_{1 \leq \alpha \leq d}, \forall i, j=1, \ldots, n .
$$

System (1.11)-(1.13) is well-posed if $u^{0}$ is regular enough:

Proposition 7. If $u^{0} \in W^{2, \infty}(\Omega)$, there exists a unique solution $\bar{u}^{1} \in H^{1}(\Omega)$ of (1.11)(1.13). 
Proof. The main point is to show that the boundary data belongs to $H^{1 / 2}(\partial \Omega)$, i.e. there exists a $U^{1} \in H^{1}(\Omega)$ such that

$$
U^{1}=\Gamma^{k} \partial_{n} u^{0}, \quad x \in \partial \Omega \cap K^{k}, 1 \leq k \leq N .
$$

Afterwards, introducing $v^{1}=u^{1}-U^{1}$, one obtains an elliptic problem with a homogeneous boundary condition and an $H^{-1}(\Omega)$ source term. It has a unique variational solution, which yields well-posedness for (1.11)-(1.13).

The difficulty is the lack of regularity near the edges and vertices of $\Omega$. When $d=2$, the situation is easier. Let $O$ be a vertex. We can assume, up to reindexing the hyperplanes, that $O$ belongs to $H^{1}$ and $H^{2}$. Then one can even find a constant matrix $G=\left(G^{1}, G^{2}\right) \in$ $M_{n}(\mathbb{R}) \times M_{n}(\mathbb{R})$ such that in the vicinity of 0 ,

$$
U^{1}:=G^{\alpha} \partial_{\alpha} u^{0}=\Gamma^{k} \partial_{n} u^{0}, \quad x \in \partial \Omega \cap K^{k}, k=1,2 .
$$

Indeed, condition (3.3) reads

$$
G^{\alpha} n_{\alpha}^{k}=\Gamma^{k}, \quad k=1,2 .
$$

Thus, to prove the existence of $G$, it is enough to show that the linear mapping

$$
M_{n}(\mathbb{R}) \times M_{n}(\mathbb{R}) \rightarrow M_{n}(\mathbb{R}) \times M_{n}(\mathbb{R}), \quad G \mapsto\left(G^{\alpha} n_{\alpha}^{1}, G^{\alpha} n_{\alpha}^{2}\right),
$$

is surjective. This follows from its straightforward injectivity. Note that in this case, only $H^{2}$ regularity of $u^{0}$ is needed.

Note also that the previous reasoning extends directly to the case of an edge (that is, the intersection of two hyperplanes) in dimension $d=3$. Let finally $O$ be a vertex of $\Omega \subset \mathbb{R}^{3}$, belonging to $M$ sides supported by $H^{1}, \ldots, H^{M}$. Let us consider a plane $H$ near 0 , transverse to the $M$ sides. It intersects $\Omega$ along a two-dimensional polygon $\tilde{\Omega}$. Locally near 0 , we can describe $\Omega$ by spherical type coordinates, that is,

$$
\Omega=\{r s: 0<r<\delta, s \in \tilde{\Omega}\} .
$$

Applying the results of the case $d=2$, we can find a smooth function

$$
G=\left(G^{1}, G^{2}\right): \tilde{\Omega} \rightarrow M_{n}(\mathbb{R}) \times M_{n}(\mathbb{R})
$$

satisfying

$$
\sum_{\alpha=1}^{2} G^{\alpha}(x) \cdot n_{\alpha}^{k}=\Gamma_{k}, \quad x \in K^{k} \cap \partial \tilde{\Omega}, 1 \leq k \leq d^{\prime} .
$$

Note that $G$ is constant near each vertex of $\tilde{\Omega}$. Back to the domain $\Omega$, we define the lift of the boundary data as

$$
U^{1}(x)=U^{1}(t s):=\sum_{\alpha=1}^{2} G^{\alpha}(s) \partial_{x_{\alpha}} u^{0}(t s) .
$$

Using the fact that $u^{0} \in W^{2, \infty}$ and $\left.\nabla u^{0}\right|_{t=0}=0$, one can easily see that $U^{1} \in H^{1}(\Omega)$. This ends the proof of Proposition 7. 
With the corrections $u^{1}$ and $u^{2}$ at hand, we will be able to prove the energy estimate (1.8), under the assumptions (A0) and (A1). Let us discuss a little these regularity requirements. Again, the main point is the irregularity of $\Omega$, which limits the smoothing effect of the elliptic operator $\nabla \cdot A^{0} \nabla$. Elliptic theory for such polygonal domains has been the subject of many papers. We refer to the textbooks [10,9].

Broadly, for an arbitrary smooth $f$ in (1.3), one cannot expect $H^{s}$ regularity for $u^{0}$ when $s>2$. For the assumption (A0) to hold, $f$ must satisfy some compatibility conditions. These compatibility conditions do not take a simple form, even for a scalar equation $(n=1)$ in dimension 2. For instance, except in the case where the angles of the polygon are of the type $\omega=\pi / n, n \in \mathbb{N}$, these conditions are not local near the vertices. We refer to [10] for details. From this point of view, assumption (A0) is restrictive.

We stress, however, that if $u^{0}$ is regular enough, assumption (A1) is quite natural. For instance, if $n=1, d=2$, and $u^{0} \in H^{4}(\Omega)$, then $U^{1} \in H^{3}(\Omega) \cap C^{1}(\bar{\Omega})$ where $U^{1}$ is the lift of the boundary data built in the previous proposition. As a result, $v^{1}=u^{1}-U^{1}$ satisfies an elliptic equation with constant coefficients, homogeneous boundary condition and source term in $H^{1}$. The $H^{2} \cap C^{1}$ regularity of $v^{1}$ then follows from standard theory for the Laplace equation in polygonal domains.

Let us stress again that by the same theory, we do not expect $u^{1}$ to be in $H^{s}(\Omega)$ with $s>2$. In other words, we do not know if the compatibility conditions imposed on $f$ should be satisfied by the source term in the equation for $v^{1}$. We pay attention to this in the next section, where we try to use as little regularity of $u^{0}$ and $u^{1}$ as possible. From now on, we assume (A0) and (A1).

\subsection{Outline of the proof}

For $i=1,2$, let $u^{i}=u^{i}(x, y)$ be as in the previous subsection, and let $u_{b l}^{i, \varepsilon}$ be the solutions of

$$
\left\{\begin{aligned}
-\nabla \cdot A(x / \varepsilon) \nabla u_{b l}^{i, \varepsilon}=0, & x \in \Omega, \\
u_{b l}^{i, \varepsilon}=-u^{i}(x, x / \varepsilon), & x \in \partial \Omega .
\end{aligned}\right.
$$

In the next subsections we shall prove the following error estimates:

1. "Global error estimate":

$$
\begin{aligned}
& \left\|e^{\varepsilon}\right\|_{H^{1}(\Omega)}=O\left(\varepsilon^{2}\right), \\
& e^{\varepsilon}:=u^{\varepsilon}-u^{0}(x)-\varepsilon u^{1}(x, x / \varepsilon)-\varepsilon^{2} u^{2}(x, x / \varepsilon)-\varepsilon u_{b l}^{1, \varepsilon}(x)-\varepsilon^{2} u_{b l}^{2, \varepsilon}(x) .
\end{aligned}
$$

2. "Boundary error estimate":

$$
\left\|e_{b l}^{\varepsilon}\right\|_{L^{2}(\Omega)}=O(\varepsilon), \quad e_{b l}^{\varepsilon}:=u_{b l}^{1, \varepsilon}-\sum_{k=1}^{N} v_{b l}^{k}(x, x / \varepsilon)+\varepsilon u_{b l}^{2, \varepsilon}
$$

where $v_{b l}^{k}(x, y)$ is the solution of (2.1) built in the previous section. 
Before we establish these bounds, let us show how they imply Theorem 1 . Let $\omega \Subset \Omega$. By the "global error estimate", we get

$$
\begin{aligned}
& \left\|u^{\varepsilon}-u^{0}(x)-\varepsilon u^{1}(x, x / \varepsilon)-\varepsilon^{2} u^{2}(x, x / \varepsilon)\right\|_{H^{1}(\omega)} \\
& \leq C \varepsilon^{2}+\left\|\sum_{k=1}^{N} v_{b l}^{k}(x, x / \varepsilon)\right\|_{H^{1}(\omega)}+\varepsilon\left\|e_{b l}^{\varepsilon}\right\|_{H^{1}(\omega)} .
\end{aligned}
$$

By our choice of $\bar{u}^{1}$, the boundary layer terms $v_{b l}^{k}(x, y)$ are fast decreasing to zero as the normal coordinate $y \cdot n^{k}-c^{k} / \varepsilon$ goes to $+\infty$, uniformly in $x$ and $\varepsilon$. The same holds for their derivatives (cf. Proposition 5). Precisely,

$$
\left\|\sum_{k=1}^{N} v_{b l}^{k}(x, x / \varepsilon)\right\|_{H^{s}\left(\omega^{\prime}\right)}=O\left(\varepsilon^{m}\right), \quad \forall s \leq 2, \forall m, \forall \omega^{\prime} \Subset \Omega .
$$

Then $e_{b l}^{\varepsilon}$ satisfies

$$
\nabla \cdot A(x / \varepsilon) \nabla e_{b l}^{\varepsilon}=r_{b l}^{\varepsilon}, \quad r_{b l}^{\varepsilon}:=-\nabla \cdot A(x / \varepsilon) \nabla \sum_{k=1}^{N} v_{b l}^{k}(x, x / \varepsilon), \quad x \in \Omega .
$$

Let $0 \leq \varphi(x) \leq 1$ be compactly supported in $\Omega$, with $\varphi=1$ in $\omega$. A standard energy estimate yields

$$
\int_{\Omega} \varphi^{2} A(x / \varepsilon) \nabla e_{b l}^{\varepsilon} \cdot \nabla e_{b l}^{\varepsilon} d x=-2 \int_{\Omega} \varphi e_{b l}^{\varepsilon} \cdot\left(A(x / \varepsilon) \nabla e_{b l}^{\varepsilon} \cdot \nabla \varphi\right) d x+\int_{\Omega} r_{b l}^{\varepsilon} \cdot \varphi^{2} e_{b l}^{\varepsilon} .
$$

Using the decay properties (3.5), the remainder term satisfies $\left\|r_{b l}^{\varepsilon}\right\|_{L^{2}\left(\omega^{\prime}\right)}=O\left(\varepsilon^{m}\right)$ for all $s, m$, and for any $\omega^{\prime} \Subset \Omega$ containing the support of $\varphi$. Thus, the above inequality implies

$$
\left\|e_{b l}^{\varepsilon}\right\|_{H^{1}(\omega)} \leq C\left\|e_{b l}^{\varepsilon}\right\|_{L^{2}(\Omega)}+C_{m} \varepsilon^{m}, \quad \forall m .
$$

Thus, we get

$$
\left\|u^{\varepsilon}-u^{0}(x)-\varepsilon u^{1}(x, x / \varepsilon)-\varepsilon^{2} u^{2}(x, x / \varepsilon)\right\|_{H^{1}(\omega)} \leq C\left(\varepsilon^{2}+\varepsilon\left\|e_{b l}^{\varepsilon}\right\|_{L^{2}(\Omega)}\right) .
$$

Combining this bound with the "boundary error estimate", we obtain (1.8), which ends the proof of Theorem 1. Actually, we have the following improved estimate which accounts for the homogenized boundary layer:

Corollary 8. Under the assumptions of Theorem 1, we also have the following global estimate:

$$
\left\|u^{\varepsilon}-u^{0}(x)-\varepsilon u^{1}(x, x / \varepsilon)-\varepsilon \sum_{k=1}^{N} v_{b l}^{k}(x, x / \varepsilon)\right\|_{L^{2}(\Omega)} \leq C \varepsilon^{2} .
$$

We point out that the difference between Theorem 1 and Corollary 8 is that Theorem 1 justifies the term $u^{2}(x, y)$ in the expansion since it gives an $H^{1}$ estimate whereas Corollary 8 justifies the boundary layer behavior since it holds up to the boundary. Of course, it only holds in $L^{2}$. 


\subsection{Global energy estimate}

This subsection is devoted to the proof of an $O\left(\varepsilon^{2}\right)$ estimate for $e^{\varepsilon}$ in $H^{1}(\Omega)$. We have

$$
-\nabla \cdot A(\cdot / \varepsilon) \nabla e^{\varepsilon}=r^{\varepsilon}, \quad x \in \Omega,\left.\quad e^{\varepsilon}\right|_{\partial \Omega}=0,
$$

where the remainder term $r^{\varepsilon}$ is given by

$$
\begin{aligned}
r^{\varepsilon}(x):= & \varepsilon \nabla_{x} \cdot\left(A \nabla_{x} \widetilde{u^{1}+A} \nabla_{y} u^{2}\right)(x, x / \varepsilon)+\varepsilon \nabla_{y} \cdot\left(A \nabla_{x} u^{2}\right)(x, x / \varepsilon) \\
& +\varepsilon^{2} \nabla_{x} \cdot\left(A \nabla_{x} u^{2}\right)(x, x / \varepsilon),
\end{aligned}
$$

with the tilde denoting the oscillating part (with zero average with respect to $y$ ). As the source term is a priori of order $\varepsilon$, one cannot obtain an $O\left(\varepsilon^{2}\right)$ bound straightforwardly. To gain extra powers of $\varepsilon$, a standard trick is then to introduce a field $W=W(x, y)$ such that

$$
\nabla_{y} \cdot W=\nabla_{x} \cdot\left(A \nabla_{x} \widetilde{u^{1}+A} \nabla_{y} u^{2}\right) .
$$

Note that if $W$ satisfies this relation, setting

$$
V(x, y)=W(x, y)+A(y) \nabla_{x} u^{2}(x, y),
$$

we can write

$$
\begin{aligned}
r^{\varepsilon}(x) & =\varepsilon \nabla_{y} \cdot W(x, x / \varepsilon)++\varepsilon \nabla_{y} \cdot\left(A \nabla_{x} u^{2}\right)(x, x / \varepsilon)+\varepsilon^{2} \nabla_{x} \cdot\left(A \nabla_{x} u^{2}\right)(x, x / \varepsilon) \\
& =\varepsilon \nabla_{y} \cdot V(x, x / \varepsilon)+\varepsilon^{2} \nabla_{x} \cdot\left(A \nabla_{x} u^{2}\right)(x, x / \varepsilon) \\
& =\varepsilon^{2} \nabla \cdot[V(\cdot, \cdot / \varepsilon)](x)-\varepsilon^{2} \nabla_{x} \cdot W(x, x / \varepsilon) .
\end{aligned}
$$

This last expression is formally enough to derive an $O\left(\varepsilon^{2}\right)$ bound. But there is a regularity issue. The r.h.s. in (3.9) involves a priori three derivatives of $u^{0}$ and two derivatives of $\bar{u}^{1}$. By (A0)-(A1), if we do not choose the solution $W$ of (3.9) carefully, it will only be $L^{2}$ with respect to $x$. That will not be enough to control the last term in the above expression for $F^{\varepsilon}$.

Inspired by ideas of Bensoussan, Lions and Papanicolaou [6], we notice that, as

$$
\nabla_{y} \cdot\left(A \nabla_{x} u^{0}+A \nabla_{y} u^{1}\right)=0,
$$

we can write

$$
A \nabla_{x} \widetilde{u^{0}+A} \nabla_{y} u^{1}=\operatorname{curl}_{y} \psi
$$

for some $\psi=\psi(x, y)$ with zero average with respect to $y$. By the assumptions on $u^{0}, u^{1}$, the field $\psi$ is smooth with respect to $y$ and has $H^{2}$ regularity with respect to $x$. Then, by construction of $u^{2}$,

$$
\nabla_{y} \cdot\left(A \nabla_{x} u^{1}+A \nabla_{y} u^{2}\right)=-\nabla_{x} \cdot\left(A \nabla_{x} \widetilde{u^{0}+A} \nabla_{y} u^{1}\right)=-\nabla_{x} \cdot \operatorname{curl}_{y} \psi=\nabla_{y} \cdot \operatorname{curl}_{x} \psi .
$$

Again, this implies that there exists $\phi=\phi(x, y)$ with zero average in $y$ such that

$$
A \nabla_{x} \widetilde{u^{1}+A} \nabla_{y} u^{2}-\operatorname{curl}_{x} \psi=\operatorname{curl}_{y} \phi .
$$


The field $\phi$ is smooth with respect to $y$ and has $H^{1}$ regularity with respect to $x$. Finally, we get

$$
\nabla_{x} \cdot\left(A \nabla_{x} \widetilde{u^{1}+A} \nabla_{y} u^{2}\right)=\nabla_{x} \cdot\left(\operatorname{curl}_{x} \psi+\operatorname{curl}_{y} \phi\right)=\nabla_{x} \cdot \operatorname{curl}_{y} \phi=-\nabla_{y} \cdot \operatorname{curl}_{x} \phi .
$$

Thus, we can set

$$
W(x, y)=-\operatorname{curl}_{x} \phi(x, y)
$$

which is smooth with respect to $y$ and has $L^{2}$ regularity with respect to $x$. The key point is that $\nabla_{x} \cdot W=0$, so that there is no lack of regularity.

From these considerations, it follows easily that

$$
\left\|r^{\varepsilon}\right\|_{H^{-1}} \leq \alpha \varepsilon^{2}
$$

with a constant $\alpha$ depending only on the $H^{2}$ norm of $u^{1}$, and the $H^{3}$ norm of $u^{0}$. Back to (3.7), a simple energy estimate gives the $O\left(\varepsilon^{2}\right)$ bound.

\subsection{Boundary layer estimate}

This subsection is devoted to the homogenization of the system

$$
\left\{\begin{array}{rr}
-\nabla \cdot A(\cdot / \varepsilon) \nabla u_{b l}^{\varepsilon}=0, & x \in \partial \Omega, \\
u_{b l}^{\varepsilon}=-u^{1}(x, x / \varepsilon)-\varepsilon u^{2}(x, x / \varepsilon), & x \in \partial \Omega .
\end{array}\right.
$$

which is satisfied by $u_{b l}^{\varepsilon}:=u_{b l}^{1, \varepsilon}+\varepsilon u_{b l}^{2, \varepsilon}$. We expect $u_{b l}^{\varepsilon}$ to have an expansion of the type

$$
u_{b l}^{\varepsilon} \approx \sum_{k=1}^{N}\left(v_{b l}^{k}(x, x / \varepsilon)+\varepsilon w_{b l}^{k}(x, x / \varepsilon)\right)
$$

where $v_{b l}^{k}=v_{b l}^{k}(x, y), w_{b l}^{k}=w_{b l}^{k}(x, y)$ are defined on the half-space $\Omega^{\varepsilon, k}$ (cf. Section 2).

Plugging the expansion in the system satisfied by $u_{b l}^{\varepsilon}$, one finds that $v_{b l}^{k}$ satisfies the system (2.1). The well-posedness and qualitative properties of this system have already been discussed. By our choice of $\bar{u}^{1}$, the solution $v_{b l}^{k}$ converges to 0 as $y \cdot n_{k}-c^{k} / \varepsilon \rightarrow$ $+\infty$, with a decay rate better than any power of $\left|y \cdot n_{k}-c^{k} / \varepsilon\right|$.

The next order term $w_{b l}^{k}$ satisfies formally:

$$
\begin{gathered}
\left\{\begin{aligned}
-\nabla_{y} \cdot A \nabla_{y} w_{b l}^{k}=f^{k}, & y \in \Omega^{\varepsilon, k}, \\
w_{b l}^{k}=-u_{2}(x, y), & y \in \partial \Omega^{\varepsilon, k},
\end{aligned}\right. \\
f^{k}:=\nabla_{x} \cdot A \nabla_{y} v_{b l}^{k}+\nabla_{y} \cdot A \nabla_{x} v_{b l}^{k} .
\end{gathered}
$$

Remark that, by decay properties of $v_{b l}^{k}, f^{k}$ goes rapidly to zero as $y \cdot n_{k}-c^{k} / \varepsilon \rightarrow+\infty$. System (3.11) is of course very similar to (2.1), and can be solved in a similar manner, taking advantage of a quasiperiodic setting. Proceeding exactly as in Section 2, it amounts to solving a problem of the form (2.7), with an $H$ which is not anymore of compact support, but satisfies $t^{m} \partial_{\theta}^{\alpha} \partial_{t}^{k} H \in L^{2}$ for all $m, \alpha, k$. The arguments for well-posedness and convergence far from the boundary extend easily to this setting. In particular, the 
conclusions of Propositions 4 and 5 are still valid. Hence, one can find $w_{b l}^{k}=w_{b l}^{k}(x, y)$ solving (3.11), that converges fast to some $w^{k, \infty}(x)$ as $y \cdot n^{k}-c^{k} / \varepsilon \rightarrow+\infty$. Note that $w_{b l}^{k}$ involves linearly second order derivatives of $u^{0}$, and first order derivatives of $\bar{u}^{1}$, so that it has $H^{1} \cap C^{0}$ regularity with respect to $x$.

Our goal is to derive an $O(\varepsilon)$ bound in $L^{2}(\Omega)$ for $\tilde{e}_{b l}^{\varepsilon}:=e_{b l}^{\varepsilon}-\varepsilon \sum w_{b l}^{k}(x, x / \varepsilon)$. As

$$
\left\|w_{b l}^{k}(x, x / \varepsilon)\right\|_{L^{2}(\Omega)}=O(1)
$$

the "boundary error estimate" will follow, concluding the proof of Theorem 1. The field $\tilde{e}_{b l}^{\varepsilon}$ satisfies

$$
-\nabla \cdot A(\cdot / \varepsilon) \nabla \tilde{e}_{b l}^{\varepsilon}=r_{b l}^{\varepsilon}, \quad x \in \Omega,\left.\quad \tilde{e}_{b l}^{\varepsilon}\right|_{\partial \Omega}=\varphi_{b l}^{\varepsilon},
$$

where

$$
\begin{aligned}
r_{b l}^{\varepsilon} & :=\sum \nabla_{x} \cdot\left(A \nabla_{x} v_{b l}^{k}+A \nabla_{y} w_{b l}^{k}\right)(x, x / \varepsilon)+\varepsilon \sum \nabla \cdot\left(A \nabla_{x} w_{b l}^{k}(\cdot, \cdot / \varepsilon)\right)(x), \\
\varphi_{b l}^{\varepsilon} & :=-u^{1}(x, x / \varepsilon)-\varepsilon u^{2}(x, x / \varepsilon)-\left.\sum\left(v_{b l}^{k}(x, x / \varepsilon)+\varepsilon w_{b l}^{k}(x, x / \varepsilon)\right)\right|_{\partial \Omega} .
\end{aligned}
$$

Control of the source term. The source term $r_{b l}^{\varepsilon}$ is made of two terms. The second term on the r.h.s. of (3.12) is of the type $\varepsilon \nabla \cdot R^{\varepsilon}$ where $\left\|R^{\varepsilon}\right\|_{L^{2}(\Omega)} \leq C$.

The first term on the r.h.s. of (3.12) reads $\sum r^{k}(x, x / \varepsilon)$ for some $r^{k}=r^{k}(x, y)$ built from $\nabla_{x} v_{b l}^{k}$ and $\nabla_{y} w_{b l}^{k}$. By properties of these boundary layer profiles, the field $r^{k}$ has $L^{2}$ regularity in $x$, is smooth in $y$, and goes to zero as $y \cdot n^{k}-c^{k} / \varepsilon \rightarrow+\infty$, faster than any power of $y \cdot n^{k}-c^{k} / \varepsilon$ uniformly in $x$ and $\varepsilon$. For any $e \in H_{0}^{1}(\Omega)$, we have

$$
\begin{aligned}
\left|\int_{\Omega} r^{k}(x, x / \varepsilon) \cdot e(x) d x\right| & \leq \int_{\Omega}\left|r_{k}\right|(x, x / \varepsilon) d(x, \partial \Omega) \frac{|e|}{d(x, \partial \Omega)} d x \\
& \leq \int_{\Omega}\left|r_{k}\right|(x, x / \varepsilon) d\left(x, K^{k}\right) \frac{|e|}{d(x, \partial \Omega)} d x \\
& \leq \int_{\Omega}\left|r_{k}\right|(x, x / \varepsilon)\left|x \cdot n^{k}-c^{k}\right| \frac{|e|}{d(x, \partial \Omega)} d x \\
& \leq \varepsilon \int_{y} \sup _{y}\left|r^{k}(x, y)\right|\left|y \cdot n^{k}-c^{k} / \varepsilon\right| \frac{|e|}{d(x, \partial \Omega)} d x \\
& \leq C \varepsilon\|\nabla e\|_{L^{2}(\Omega)}
\end{aligned}
$$

where the last inequality stems from Cauchy-Schwarz' and Hardy's inequalities.

Gathering these bounds gives

$$
\left\|r_{b l}^{\varepsilon}\right\|_{H^{-1}(\Omega)} \leq C \varepsilon
$$

Control of the boundary term. We will prove that

$$
\left\|\varphi_{b l}^{\varepsilon}\right\|_{W^{1-1 / p, p}(\partial \Omega)} \leq C(p) \varepsilon, \quad \forall p<2 .
$$

Before that, let us show how this implies the bound we want on $\tilde{e}_{b l}^{\varepsilon}$. First, it allows us to introduce a field $\phi^{\varepsilon}$ satisfying, for all $p<2$,

$$
\phi^{\varepsilon} \in W^{1, p}(\Omega),\left.\quad \phi^{\varepsilon}\right|_{\partial \Omega}=\varphi_{b l}^{\varepsilon}, \quad\left\|\phi^{\varepsilon}\right\|_{W^{1, p}(\Omega)}=O(\varepsilon) .
$$


The remaining term $e^{\varepsilon}=\tilde{e}_{b l}^{\varepsilon}-\phi^{\varepsilon}$ satisfies

$\nabla \cdot\left(A(x / \varepsilon) \nabla e^{\varepsilon}\right)=F^{\varepsilon}$ in $\Omega,\left.\quad e^{\varepsilon}\right|_{\partial \Omega}=0, \quad F^{\varepsilon}=r_{b l}^{\varepsilon}-\nabla \cdot\left(A(x / \varepsilon) \nabla \phi^{\varepsilon}\right) \in W^{-1, p}(\Omega)$.

We can now apply general results of Meyers [12] on elliptic equations in divergence form with bounded coefficients. These results extend straightforwardly to elliptic systems (i.e. when $n>1$ ). As a result, there exists $p_{m}<2$ such that for all $p_{m}<p<2$, $e^{\varepsilon}$ satisfies

$$
\left\|e^{\varepsilon}\right\|_{W^{1, p}(\Omega)} \leq C(p)\left\|F^{\varepsilon}\right\|_{W^{-1, p}(\Omega)} \leq C(p) \varepsilon
$$

combining (3.14) and the estimate on $\phi^{\varepsilon}$. The $L^{2}$ estimate on $e^{\varepsilon}$, and then on $\tilde{e}_{b l}^{\varepsilon}$, follows from Sobolev imbedding.

Hence, the last step is to obtain (3.15). We first focus on one part of $\varphi_{b l}^{\varepsilon}$, namely

$$
\varphi_{v}: x \mapsto-u^{1}(x, x / \varepsilon)-\sum_{k} v_{b l}^{k}(x, x / \varepsilon) .
$$

We shall prove that

$$
\left\|\varphi_{v}\right\|_{W^{1-1 / p, p}(\partial \Omega)}=O(\varepsilon), \quad \forall p<2 .
$$

By construction of the $v_{b l}^{k}$ 's, one can decompose

$$
\varphi_{v}(x)=V(x, x / \varepsilon) \nabla u^{0}(x):=\left(-\chi(x / \varepsilon)+\sum_{k} V^{k}(x / \varepsilon)\right) \nabla u^{0}(x),
$$

where

$V=V^{\alpha}(y), \quad \chi=\chi^{\alpha}(y), \quad V^{k}=V^{k, \alpha}(y) \in M_{n}(\mathbb{R}), \quad \alpha=1, \ldots, d, k=1, \ldots, N$,

denote as usual families of matrix fields. Note that $\chi$ is the solution of the cell problem (1.4). By construction of the boundary layer profiles, $v_{b l}^{k}$ and its derivatives go to zero uniformly as $y \cdot n^{k}-c^{k} / \varepsilon \rightarrow+\infty$, faster than any negative power of $y \cdot n^{k}-c^{k} / \varepsilon$. Moreover, for any $k$,

$$
\left.\varphi_{v}\right|_{\partial \Omega \cap K^{k}}=-\sum_{j \neq k} V^{j}(x / \varepsilon) \nabla u^{0}(x)
$$

Let $\psi$ be a smooth function on $\partial \Omega$, compactly supported outside a neighborhood of the edges and vertices of $\Omega$. The above remarks lead to: for all $p<2$,

$$
\left\|\psi \varphi_{v}\right\|_{W^{1-1 / p, p}(\partial \Omega)} \leq\left\|\psi \varphi_{v}\right\|_{H^{1 / 2}(\partial \Omega)}=C_{s, m} \varepsilon^{m}\left\|\nabla u^{0}\right\|_{H^{1 / 2}(\partial \Omega)} \leq C_{s, m}^{\prime} \varepsilon^{m}, \quad \forall m, s .
$$

Hence, the main problem in establishing the $O(\varepsilon)$ bound comes from the edges and vertices of the polygon. In particular, we will need to use cancellation properties of $\nabla u^{0}$ there.

Let us first consider the case $n=2$. Let $O$ be a vertex of $\Omega$. We introduce polar coordinates $r=r(x), \theta=\theta(x)$, centered at $O$. Let $\psi$ be a smooth function supported this 
time in the vicinity of $O$ in $\partial \Omega$. We recall the standard estimate: for all $f, g \in L^{\infty}(\partial \Omega) \cap$ $W^{1-1 / p, p}(\partial \Omega)$,

$$
\|f g\|_{W^{1-1 / p, p}(\partial \Omega)} \leq C\left(\|f\|_{L^{\infty}(\partial \Omega)}\|g\|_{W^{1-1 / p, p}(\partial \Omega)}+\|g\|_{L^{\infty}(\partial \Omega)}\|f\|_{W^{1-1 / p, p}(\partial \Omega)}\right) .
$$

Hence, we deduce

$$
\begin{aligned}
\left\|\psi^{2} \varphi_{v}\right\|_{W^{1-1 / p, p}(\partial \Omega)} \leq & \left\|\psi \frac{\nabla u^{0}}{r}\right\|_{W^{1-1 / p, p}(\partial \Omega)}\|\psi r V(\cdot / \varepsilon)\|_{L^{\infty}(\partial \Omega)} \\
& +\left\|\psi \frac{\nabla u^{0}}{r}\right\|_{L^{\infty}(\partial \Omega)}\|\psi r V(\cdot / \varepsilon)\|_{W^{1-1 / p, p}(\partial \Omega)} .
\end{aligned}
$$

We emphasize that

$$
\psi \frac{\nabla u^{0}}{r} \in L^{\infty}(\partial \Omega) \cap W^{1-1 / p, p}(\partial \Omega), \quad \forall p<2 .
$$

Indeed, as $u^{0}$ satisfies a Dirichlet condition at $\partial \Omega, \nabla u^{0}$ cancels at the vertex $O$, and Taylor's formula gives

$$
\frac{\nabla u^{0}(x)}{r}=\frac{x}{|x|} \cdot \int_{0}^{1} \nabla \nabla u^{0}(t x) d t .
$$

By assumption (A0), it clearly belongs to $L^{\infty}(\partial \Omega)$ and to $W^{1, p}(\Omega)$, hence to $W^{1-1 / p, p}(\partial \Omega)$. Note however that it does not belong a priori to $H^{1 / 2}(\partial \Omega)$. That is why we consider $L^{p}$ spaces for $p<2$ and use the Meyers theorem.

It remains to control the function $r V(\cdot / \varepsilon)$ in the vicinity of $O$ in $\partial \Omega$. This vertex belongs to two sides, say $K^{1} \cap \partial \Omega$ and $K^{2} \cap \partial \Omega$. We can always assume that $\theta=0$ corresponds to $K^{1}$ and $\theta=\omega$ corresponds to $K^{2}$. Note that by convexity, $0<\omega<\pi$. For $j \neq 1$, by properties of the boundary layer profiles,

$$
\left\|\psi r V^{j}(\cdot / \varepsilon)\right\|_{H^{s}\left(\partial \Omega \cap K^{2}\right)}=O\left(\varepsilon^{m}\right), \quad \forall m, s .
$$

We can therefore neglect such terms. Then

$$
\begin{aligned}
\|\psi r V(\cdot / \varepsilon)\|_{L^{\infty}\left(\partial \Omega \cap K^{2}\right)} & \leq C \sup _{r>0} r\left|V^{1}(r \cos \omega / \varepsilon, r \sin \omega / \varepsilon)\right| \\
& \leq c \frac{\varepsilon}{\sin \omega} \sup _{y}\left|y_{2}\right|\left|V^{1}\left(y_{1}, y_{2}\right)\right| \leq C \varepsilon .
\end{aligned}
$$

Similarly,

$$
\|\psi r V(\cdot / \varepsilon)\|_{L^{p}\left(\partial \Omega \cap K^{2}\right)} \leq C \frac{\varepsilon^{1+1 / p}}{(\sin \omega)^{1 / p}} \sup _{y_{1}}\left(\int_{0}^{+\infty}\left|V^{1}\left(y_{1}, y_{2}\right)\right|^{p} d y_{2}\right)^{1 / p} \leq C^{\prime} \varepsilon^{1+1 / p} .
$$

Applying the same reasoning to the tangential derivatives, we get

$$
\|\psi r V(\cdot / \varepsilon)\|_{H^{1}\left(\partial \Omega \cap K^{2}\right)} \leq C^{\prime} \varepsilon^{1 / p} .
$$


We can of course proceed in a similar way with the other hyperplane $K^{1}$, and we end up with

$$
\begin{gathered}
\|\psi r V(\cdot / \varepsilon)\|_{L^{\infty}(\partial \Omega)}=O(\varepsilon), \\
\|\psi r V(\cdot / \varepsilon)\|_{L^{1}(\partial \Omega)}=O\left(\varepsilon^{1+1 / p}\right), \quad\|\psi r V(\cdot / \varepsilon)\|_{W^{1, p}(\partial \Omega)}=O\left(\varepsilon^{1 / p}\right) .
\end{gathered}
$$

By interpolation of the last two inequalities, we get

$$
\|\psi r V(\cdot / \varepsilon)\|_{W^{1-1 / p, p}(\partial \Omega)}=O\left(\varepsilon^{2 / p}\right),
$$

which gives the bound we want for the case $d=2$.

When $d=3$, the computations are almost the same. We have to distinguish between the case of an edge and the case of a vertex.

- In the neighborhood of an edge, but far from a vertex, one can use locally cylindrical coordinates $(r, \theta, z)$, where $r=0$ corresponds to the edge, $z$ is the variable along the edge, and $\theta$ is the angular variable. Again, the edge is the intersection of two hyperplanes $K^{1}$ and $K^{2}$, with $\theta=0$ corresponding to $K^{1}$, whereas $\theta=\omega$ corresponds to $K^{2}$. The computation is exactly the same as for $d=2$, and we leave the details to the reader.

- In the neighborhood of a vertex $O$, one can use spherical type coordinates. Precisely, we consider a plane $H$ near $O$, transverse to the sides that contain $O$. Its intersection with $\Omega$ is a two-dimensional polygon $\tilde{\Omega}$. We describe $\partial \Omega$ near $O$ by the coordinates $x=r s, r>0, s \in \partial \tilde{\Omega}$. We use this time the decomposition

$$
\psi^{2} \varphi_{v}=(\psi|r s| V(x / \varepsilon))\left(\psi \frac{\nabla u^{0}(x)}{|r s|}\right)
$$

for $\psi=\psi(x)$ a function compactly supported near $O$. Thanks to (3.16), we must again evaluate $(\psi|r s| V)(x / \varepsilon)$. For instance,

$$
\|\psi|r s| V(x / \varepsilon)\|_{L^{\infty}(\partial \Omega)} \leq \varepsilon \sup _{\sigma \in(r / \varepsilon) \partial \tilde{\Omega}}|\sigma| V(\sigma) \leq C \varepsilon .
$$

The treatments of the $L^{p}$ norm and $W^{1, p}$ norm are similar. We end up with

$$
\|\psi|r s| V(x / \varepsilon)\|_{W^{1-1 / p, p}(\partial \Omega)} \leq C \varepsilon^{2 / p},
$$

which concludes the study of $\varphi_{v}$.

To establish inequality (3.15), it remains to handle the other part of $\varphi_{b l}^{\varepsilon}$. Namely,

$$
\varphi_{w}:=-u^{2}(x, x / \varepsilon)-\sum_{k} w_{b l}^{k}(x, x / \varepsilon)
$$

should satisfy

$$
\left\|\varphi_{w}\right\|_{W^{1-1 / p, p}(\partial \Omega)}=O(1), \quad \forall p<2 .
$$


Again, by the properties of the $w_{b l}^{k}$ 's, we can write

$$
\begin{aligned}
\varphi_{w} & =\mathcal{W}(x / \varepsilon) \nabla^{2} u^{0}(x)+W(x / \varepsilon) \nabla \bar{u}^{1}(x) \\
& :=\left(-\Upsilon(x / \varepsilon)+\sum_{k} \mathcal{W}^{k}(x / \varepsilon)\right) \nabla^{2} u^{0}(x)+\left(-\chi(x / \varepsilon)+\sum_{k} W^{k}(x / \varepsilon)\right) \nabla \bar{u}^{1}(x),
\end{aligned}
$$

where

$$
\begin{array}{r}
\mathcal{W}=\mathcal{W}^{\alpha \beta}(y), \quad \Upsilon=\Upsilon^{\alpha \beta}(y), \quad \mathcal{W}^{k}=\mathcal{W}^{k, \alpha \beta}(y) \in M_{n}(\mathbb{R}), \\
\alpha, \beta=1, \ldots, d, k=1, \ldots, N,
\end{array}
$$

and

$$
\begin{array}{r}
W=W^{\alpha}(y), \quad \chi=\chi^{\alpha}(y), \quad W^{k}=W^{k, \alpha}(y) \in M_{n}(\mathbb{R}), \\
\\
\alpha=1, \ldots, d, k=1, \ldots, N .
\end{array}
$$

Note that $\Upsilon$ and $\chi$ are the same families as in (1.9) and (1.4).

Unlike the previous fields $V^{k}$, the fields $\mathcal{W}^{k}$, resp. $W^{k}$ converge to non-zero constant fields $\mathcal{W}^{k, \infty}$, resp. $W^{k, \infty}$. To overcome this difficulty, we introduce the field $\varphi_{w}^{\infty}$, defined by

$$
\varphi_{w}^{\infty}:=\sum_{j \neq k} \mathcal{W}^{j, \infty} \nabla^{2} u^{0}(x)+\sum_{j \neq k} W^{j, \infty} \nabla \bar{u}^{1}(x), \quad x \in \partial \Omega \cap K^{k} .
$$

Note that $\varphi_{w}^{\infty}$ can be decomposed into products of the type $f g$, where:

- $f$ involves either second derivatives of $u^{0}$ or first derivatives of $u^{1}$. By (A0) and (A1), it belongs to $H^{1}(\Omega) \cap C^{0}(\bar{\Omega})$.

- $g=g^{k}$ is constant on each hyperplane $K^{k}$. Direct verifications show that

$$
g \in W^{1-1 / p, p}(\partial \Omega), \quad \forall p<2 .
$$

For instance, when $d=2$, the only regularity problem lies at the vertices of $\Omega$. Let $O$ be such a vertex, belonging for instance to $K^{1} \cap K^{2}$. It is then enough to find $G \in W^{1, p}(\Omega)$ such that $\left.G\right|_{\partial \Omega}=g$ in the vicinity of $O$. As before, we consider polar coordinates $r, \theta$ centered at 0 . The angle $\theta=0$ corresponds to $K^{1}$, and $\theta=\omega$ corresponds to $H^{l}$. We take

$$
G=\left(1-\sin \left(\frac{\pi \theta}{2 \omega}\right)\right) g_{1}+g_{2} \sin \left(\frac{\pi \theta}{2 \omega}\right) \in W^{1, p}(\Omega), \quad \forall p<2 .
$$

We stress that such a field $G$ is not in $H^{1}(\Omega)$, so that considering $p<2$ is again needed. When $d=3$, the treatment is similar and left to the reader.

We deduce that $\left\|\varphi_{w}^{\infty}\right\|_{W^{1-1 / p, p}(\partial \Omega)}<+\infty$. Now, for any $k=1, \ldots, N$, and all $x \in$ $\partial \Omega \cap K^{k}$, one has

$$
\varphi_{w}(x)=\varphi_{w}^{\infty}(x)+\sum_{j \neq k}\left(\mathcal{W}^{j}(x / \varepsilon)-\mathcal{W}^{j, \infty}\right) \nabla^{2} u^{0}(x)+\left(W^{j}(x / \varepsilon)-W^{j, \infty}\right) \nabla \bar{u}^{1}(x) .
$$


Hence, up to replacing $\varphi_{w}$ by $\varphi_{w}-\varphi_{w}^{\infty}$, we can always assume that $\mathcal{W}^{k, \infty}=0$, $W^{k, \infty}=0$.

At this point, the estimate on $\varphi_{w}$ can be obtained along the same lines as the estimate on $\varphi_{v}$. As we only need an $O(1)$ bound, the situation is simpler: we do not need extra terms like $r$ (for $d=2$ ) or $r s$ (for $d=3$ ) in front of the boundary layer terms $\mathcal{W}(x, x / \varepsilon$ ) and $W(x / \varepsilon)$. In other words, we do not need any cancelation property for $\nabla^{2} u^{0}$ or $\nabla \bar{u}^{1}$. This concludes the proof of Theorem 1.

Acknowledgments. D. G-V. is supported by ANR Grant ANR-08-JCJC-0104 and N. M. is partially supported by NSF Grant DMS-0703145.

\section{References}

[1] Allaire, G., Amar, M.: Boundary layer tails in periodic homogenization. ESAIM Control Optim. Calc. Var. 4, 209-243 (1999) Zbl 0922.35014 MR 1696289

[2] Allaire, G., Conca, C.: Bloch-wave homogenization for a spectral problem in fluid-solid structures. Arch. Ration. Mech. Anal. 135, 197-257 (1996) Zbl 0857.73008 MR 1418465

[3] Allaire, G., Conca, C.: Boundary layers in the homogenization of a spectral problem in fluidsolid structures. SIAM J. Math. Anal. 29, 343-379 (1998) Zbl 0918.35018 MR 1616495

[4] Allaire, G., Conca, C.: Bloch wave homogenization and spectral asymptotic analysis. J. Math. Pures Appl. (9) 77, 153-208 (1998) Zbl 0901.35005 MR 1614641

[5] Avellaneda, M., Lin, F. H.: Compactness methods in the theory of homogenization. Comm. Pure Appl. Math. 40, 803-847 (1987) Zbl 0632.35018 MR 0910954

[6] Bensoussan, A., Lions, J.-L., Papanicolaou, G.: Asymptotic Analysis for Periodic Structures. Stud. Math. Appl. 5, North-Holland, Amsterdam (1978) Zbl 0404.35001 MR 0503330

[7] Cassels, J. W. S.: An Introduction to Diophantine Approximation. Hafner, New York, 1972. Facsimile reprint of the 1957 edition, Cambridge Tracts Math. Math. Phys. 45 Zbl 0077.04801 MR 0087708

[8] Cioranescu, D.: An Introduction to Homogenization. Oxford Univ. Press (1999) Zbl 0939.35001 MR 1765047

[9] Dauge, M.: Elliptic Boundary Value Problems on Corner Domains. Lecture Notes in Math. 1341, Springer, Berlin (1988) Zbl 0668.35001 MR 0961439

[10] Grisvard, P.: Elliptic Problems in Nonsmooth Domains. Monogr. Stud. Math. 24, Pitman (Adv. Publ. Program), Boston, MA (1985) Zbl 0695.35060 MR 0775683

[11] Jikov, V. V., Kozlov, S. M., Oleŭnik, O. A.: Homogenization of Differential Operators and Integral Functionals. Springer, Berlin (1994) Zbl 0838.35001 MR 1329546

[12] Meyers, N. G.: An $L^{p}$-estimate for the gradient of solutions of second order elliptic divergence equations. Ann. Scuola Norm. Sup. Pisa (3) 17, 189-206 (1963) Zbl 0127.31904 MR 0159110

[13] Moskow, S., Vogelius, M.: First-order corrections to the homogenised eigenvalues of a periodic composite medium. A convergence proof. Proc. Roy. Soc. Edinburgh Sect. A 127, $1263-$ 1299 (1997) Zbl 0888.35011 MR 1489436

[14] Murat, F.: Compacité par compensation. Ann. Scuola Norm. Sup. Pisa (3) 5, 489-507 (1978) Zbl 0399.46022 MR 0506997

[15] Neuss-Radu, M.: The boundary behavior of a composite material. Math. Model. Numer. Anal. 35, 407-435 (2001) Zbl 0985.35092 MR 1837078 
[16] Santosa, F., Vogelius, M.: First-order corrections to the homogenized eigenvalues of a periodic composite medium. SIAM J. Appl. Math. 53, 1636-1668 (1993) Zbl 0808.35085 MR 1247172

[17] Santosa, F, Vogelius, M.: Erratum to the paper: "First-order corrections to the homogenized eigenvalues of a periodic composite medium". SIAM J. Appl. Math. 55, 864 (1995) MR 1331590

[18] Sarkis, M., Versieux, H.: Convergence analysis for the numerical boundary corrector for elliptic equations with rapidly oscillating coefficients. SIAM J. Numer. Anal. 46, 545-576 (2008) Zbl 1176.65123 MR 2383203

[19] Tartar, L.: H-measures, a new approach for studying homogenization, oscillations and concentration effects in partial differential equations. Proc. Roy. Soc. Edinburgh Sect. A 115, 193-230 (1990) Zbl 0774.35008 MR 1069518

[20] Versieux, H. M., Sarkis, M.: Numerical boundary corrector for elliptic equations with rapidly oscillating periodic coefficients. Comm. Numer. Methods Engrg. 22, 577-589 (2006) Zbl 1109.65104 MR 2235030 